\section{The Hamilton Depression Scale- accelerator or break on antidepressant drug discovery?}

\author{
David Nutt
}

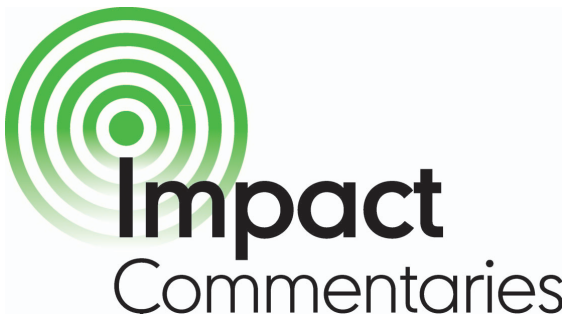

Fifty years ago Max Hamilton published the Hamilton Depression Scale (HAMD) scale for rating depression ${ }^{1}$-specifically, changes in this state that might be used to monitor treatment progress. It proved exceptionally popular being one of-if not the-most cited paper in psychiatry and propelling Max to the top of the all-time citation charts in psychiatric journals-just behind Freud.

Hamilton was born in Germany in 1912, but the family moved to England in 1915, changing their name from Himmelschein. He studied medicine at University College London (UCL), then trained in psychiatry at the Maudsley Hospital. He returned to UCL when Sir Aubrey Lewis declined to renew his contract. His talents were however recognised and encouraged at UCL, and his interest in psychometrics developed and was then further encouraged during a period working for Dennis Hill. He then moved to the University of Leeds where, working as a lecturer he developed his scale.

The reasons for its success are clear. It was an idea for its time: the field was undergoing a sea change from personalised psychotherapeutically-based interactions to diagnosis-based treatments, and although Hamilton was forever pointing out that the HAMD was not intended to be used to make the diagnosis of depression, his scale provided a structured interview that captured the essence of the illness of depression in a simple reproducible manner.

Despite not having either ideal sensitivity or specificity-there was a great deal of

Professor David Nutt, The Edmond I Safra Chair, Centre for Neuropsychopharmacology, Division of Brain Sciences, Department of Medicine, Imperial College London, Burlington-Danes Building, Hammersmith Hospital, Du Cane Road, London W12 ONN: d.nutt@imperial.ac.uk
A rating scale for depression

Author: Max Hamilton

Published: I Neurol Neurosurg

Psychiatry 1960;23:56-62

Total citations: 15882

overlap with symptoms of other diagnoses, particularly anxiety-the scale did capture the key elements of depression, and when applied to a depressed patient, it had a robustness that made it very suitable for monitoring changes in symptoms. It was easy to use and followed the pattern of enquiry that clinicians were beginning to use to elicit depression in patients. For these reasons, it became popular in early trials of antidepressants. Then as data sets accrued, it became the gold standard as it allowed the efficacy of new treatments such as the selective serotonin reuptake inhibitors to be to some extent compared with older drugs such as the tricyclic antidepressants (TCAs) and monoamine oxidase inhibitors.

This very robustness was in some senses a weakness of the scale. The field became rather ossified; depression became viewed as a syndrome that could be defined by HAMD scores and more encompassing and sensitive scales such as the Montgomery-Asberg Depression Rating Scale (MADRS) ${ }^{2}$ struggled to gain acceptance. A kind of HAMD inertia set in and is still pervasive today. For although regulators insist that they are comfortable with any validated depression rating system, many pharma companies are phobic of conducting a depression study without the HAMD, knowing that to do so will raise eyebrows and lead to a feeling that they did not have confidence in their new drug. For this reason, the HAMD, can be considered to have put something of a break on innovation in psychiatric treatment. To a certain extent its widespread use has led to the development of drugs that change the HAMD rather than necessarily optimising outcomes in depression. This has meant that drugs that act on monoamines, and so, mimic the mode of action of the prototypical antidepressants the TCAs, may have been selected over more novel agents. This may have contributed to drug failures such as with the substance $P$ antagonists.

Another more obvious weakness is that patients can exhibit major changes in scores that could mimic antidepressant effects from drugs that hit common symptoms in the scale that might not be representative of 'core' depression. Most obviously there was the question of the three sleep items-onset, middle and late insomnia; in theory a sleep-promoting agent could produce sufficiently large changes in these three items to appear antidepressant. In practice, this does not appear to have led to the licensing of hypnotics as antidepressants although there were studies with benzodiazepines that did reveal significant changes in the HAMD, to a large extent due to effects on these and the anxiety items. These concerns were expressed at the regulatory level, and in an attempt to overcome them, Per Bech and colleagues refined the HAMD to allow a 'core' depression score ${ }^{3}$ to the extracted from items that were seen as central to depression-low mood, loss of pleasure and retardation.

Nowadays, most clinical trials of antidepressants present data on this subset of items as further proof of target validity. This became a particularly pertinent issue recently with the development of agomelatine for depression. As this drug acts predominantly as a melatonin receptor agonist (with some 5HT2C receptor antagonism as well) and melatonin is involved in sleep regulation, there were concerns that agomelatine might simply affect the sleep items on the HAMD; however, this was shown not to be the case using subscales and indeed individual item analyses. ${ }^{4}$

So overall, what has been the contribution of the HAMD to psychiatry? It certainly led the way in terms of providing a simple reproducible metric of mood change to the benefit of outcome measurements of clinical treatment as well as clinical trials. Fifty years on, some would argue whether it should maintain its status as the primary outcome variable in the clinical assessment of new antidepressants. More sensitive clinician rated scales, particularly the MADRS, may have superseded it, and there is growing encouragement for the use of patient-rated scales such as the Rush Quick Inventory of Depressive Symptomatology (QIDS) ${ }^{5}$ in combination, but in defining an approach to measurement of treatment effectiveness in psychiatric disorders, the HAMD will always retain a unique place in psychiatric history.

Competing interests None. 
Provenance and peer review Commissioned; internally peer reviewed.

To cite Nutt D. I Neurol Neurosurg Psychiatry 2014;85:119-120.

Accepted 9 October 2013

J Neurol Neurosurg Psychiatry 2014;85:119-120. doi:10.1136/jnnp-2013-306984

\section{REFERENCES}

1 Hamilton M. A rating scale for depression. J Neurol Neurosurg Psychiatry 1960;23:56-62.

2 Montgomery SA, Asberg M. A new depression scale designed to be sensitive to change. $\mathrm{Br} J$ Psychiatry 1979;134:382-89.

3 Bech P, Gram LF, Dein E, et al. Quantitative rating of depressive states. Acta Psychiatr Scand 1975:51:161-70
4 Olié JP, Kasper S. Efficacy of agomelatine, a MT1/MT2 receptor agonist with 5-HT2C antagonistic properties, in major depressive disorder. Int $\mathrm{J}$

Neuropsychopharmacol 2007;10:661-73.

5 Rush AJ, Trivedi MH, Ibrahim HM, et al. The 16-Item Quick Inventory of Depressive Symptomatology (QIDS), clinician rating (QIDS-C), and self-report (QIDS-SR): a psychometric evaluation in patients with chronic major depression. Biol Psychiatry 2003;54:573-83. 